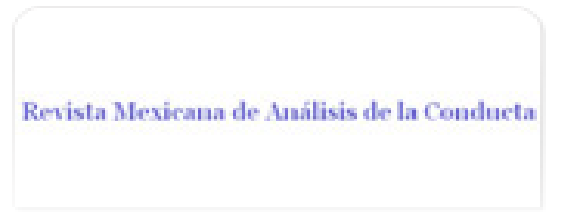

Revista Mexicana de Análisis de la Conducta ISSN: 0185-4534

editora@rmac-mx.org

Sociedad Mexicana de Análisis de la Conducta México

Valdez-Ramírez, Pablo; Ramírez-Tule, Candelaria; García-García, Aída; Talamantes-López, Javier RITMOS CIRCADIANOS EN LA EFICIENCIA PARA RESPONDER EN UNA PRUEBA DE EJECUCIÓN CONTINUA

Revista Mexicana de Análisis de la Conducta, vol. 35, núm. 1, junio, 2009, pp. 75-91

Sociedad Mexicana de Análisis de la Conducta

Guadalajara, México

Disponible en: http://www.redalyc.org/articulo.oa?id=59311416005

- Cómo citar el artículo

- Número completo

- Más información del artículo

Página de la revista en redalyc.org

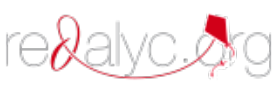

Sistema de Información Científica

Red de Revistas Científicas de América Latina, el Caribe, España y Portugal Proyecto académico sin fines de lucro, desarrollado bajo la iniciativa de acceso abierto 


\title{
RITMOS CIRCADIANOS EN LA EFICIENCIA PARA RESPONDER EN UNA PRUEBA DE EJECUCIÓN CONTINUA
}

\author{
CIRCADIAN RHYTHMS IN RESPONSE EFFICIENCY \\ ON A CONTINUOUS PERFORMANCE TEST
}

\author{
PABLO VALDEZ-RAMÍREZ, CANDELARIA RAMÍREZ-TULE, \\ AÍDA GARCÍA-GARCÍA Y JAVIER TALAMANTES-LÓPEZ1 \\ LABORATORIO DE PSICOFISIOLOGÍA, FACULTAD DE PSICOLOGÍA, \\ UNIVERSIDAD AUTÓNOMA DE NUEVO LEÓN
}

\begin{abstract}
RESUMEN
El objetivo del presente estudio fue determinar si existen variaciones circadianas en la eficiencia para responder ante estímulos con diferente frecuencia, en una tarea de ejecución continua que mide la atención sostenida. Se registraron tres mujeres voluntarias, estudiantes universitarias, de 17, 18 y 19 años de edad, durante al menos 25 horas, en condiciones controladas de laboratorio, con un protocolo de rutina constante, que permite reducir la influencia de los sincronizadores sobre los ritmos circadianos. Se registró la temperatura rectal y la ejecución en una prueba de ejecución continua, en la que se presentaron tres estímulos con las siguientes frecuencias: $70 \%, 20 \%$ y $10 \%$. Se encontraron variaciones circadianas en la temperatura rectal y en la eficiencia para responder a la tarea de ejecución continua, así como en la eficiencia para responder a todos los estímulos, independientemente de su
\end{abstract}

1. Agradecemos al Dr. Luiz Menna-Barreto por enviarnos el programa para computadora COSANA, elaborado por la Dra. Ana Amélia Benedito-Silva, por medio del cual se llevó a cabo el análisis cosinor. Agradecemos a las participantes su enorme esfuerzo y entusiasta colaboración. Correspondencia: Pablo Valdez-Ramírez, Laboratorio de Psicofisiología, Facultad de Psicología, Universidad Autónoma de Nuevo León, Mutualismo 110, Col. Mitras Centro, Monterrey, NL, 64460, México. Correo electrónico: pavaldez@yahoo.com

Recibido: 2 de Diciembre del 2008 Revisado: Enero 15 del 2009 Aceptado: Mayo 6 del 2009. 
frecuencia. En conclusión, los ritmos circadianos en la atención sostenida prevalecen a pesar de que se usen estímulos con diferente frecuencia.

Palabras Clave: Ritmos circadianos, rendimiento, tarea de ejecución continua, atención sostenida, humanos.

\begin{abstract}
The objective of this study was to determine if there are circadian variations in performance to stimuli with different frequency, in a continuous performance task that measures sustained attention. Three female undergraduate students, 17,18 and 19 years of age, were recorded during at least 25 hours in a constant routine protocol, designed to reduce the influence of synchronizers on circadian rhythms. Rectal temperature and responses on a continuous performance task were recorded. This task included three stimuli with the following frequencies: $70 \%, 20 \%$ and $10 \%$. Results showed circadian variations in rectal temperature and response efficiency to the continuous performance task and for all stimuli independently of their frequency. In conclusion, circadian rhythms in sustained attention prevail despite using stimuli with different frequencies.
\end{abstract}

Key Words: Circadian rhythms, performance, continuous performance task, sustained attention, human.

La vida surgió en un medio cíclico, ya que los movimientos de rotación y traslación de nuestro planeta producen oscilaciones en la iluminación y la temperatura. La rotación terrestre produce el día y la noche, mientras que la traslación produce las estaciones del año. Los seres vivos se adaptaron a estas condiciones generando oscilaciones regulares en su fisiología, que se conocen como ritmos biológicos (Moore-Ede, Sulzman \& Fuller, 1982). Los organismos que logran sincronizar su actividad fisiológica a las condiciones ambientales tienen mayores probabilidades de sobrevivir en un ambiente cíclico (Luce, 1971). El ritmo biológico mejor estudiado se conoce como ritmo circadiano (circa=cerca, dies=día), porque adopta un período cercano a un día (Palmer, 1976). Se han documentado variaciones circadianas en casi todos los seres vivos, desde organismos unicelulares, plantas, animales, hasta el ser humano (Koukkari \& Sothern, 2006). La mayor parte de la fisiología de los organismos presenta ritmos circadianos, un ejemplo es la temperatura corporal, la cual aumenta en el día y disminuye en la noche. Otros ejemplos son: la secreción de hormonas como el cortisol, la noradrenalina, las gonadotrofinas, la frecuencia cardiaca, la excreción urinaria de diversos electrolitos como sodio, calcio, potasio, así como la concentración de distintos neurotransmisores en el cerebro (Touitou, 1998). Los ritmos circadianos son 
estables, pero a la vez son modulados por sincronizadores, tales como: el ciclo de luz y oscuridad, el ciclo de temperatura ambiental, la disponibilidad de alimento, el ejercicio y la estimulación social. Estos ritmos se manifiestan claramente cuando se reduce la influencia de los sincronizadores, esto se logra por medio de un "protocolo de rutina constante". Este protocolo constituye una técnica fundamental para estudiar los ritmos circadianos, consiste en mantener constantes: la temperatura ambiental, la iluminación, el ingreso calórico (alimentos), la postura corporal (control del gasto calórico por medio de restricción de movimientos) y se limita la estimulación social (Hanneman, 2001).

No solamente la fisiología oscila con un periodo de 24 horas, también se han encontrado variaciones circadianas en el comportamiento de los animales (Valdez, Pérez, Galarza \& Ramírez, 1986) y del ser humano (Colquhoun, 1971). En general, se ha encontrado que la ejecución es más eficiente durante el día, mientras que empeora en la madrugada (Lavie, 1980; Carrier \& Monk, 2000). Kleitman planteó que los ritmos en la fisiología modulan la actividad de los organismos (Dement \& Kleitman, 1957). De acuerdo con la teoría de Kleitman, las oscilaciones circadianas en el metabolismo corporal producen los cambios en la ejecución. Es importante mencionar que el metabolismo corporal se refiere a todas las reacciones químicas del organismo, por lo que implica cambios en todos los procesos fisiológicos, como la temperatura corporal, así como la actividad de todos los órganos del cuerpo, incluyendo al sistema nervioso. La temperatura corporal es un índice básico del metabolismo corporal, por lo que de acuerdo con Kleitman, el nivel en la temperatura corporal permite predecir el nivel de ejecución de los organismos (Kleitman, 1963). Por otro lado, Borbély (1982) propuso que la ejecución en el tiempo es el resultado de la interacción entre los ritmos circadianos y un proceso homeostático. Este proceso se refiere al nivel de fatiga que tiene un organismo a distintas horas del día. Generalmente, al momento de despertar un organismo se encuentra listo para la acción, con un nivel máximo de ejecución; pero entre más tiempo permanezca despierto y entre mayor actividad tenga, mayor será su fatiga y somnolencia, por lo que su ejecución irá disminuyendo a través del tiempo (Åkerstedt et al., 2004). El dormir y el descanso disminuyen la somnolencia y la fatiga, por lo tanto mejoran la ejecución. La ejecución en cada momento del día depende de la suma de los valores de los procesos circadianos y homeostáticos.

En el ser humano se han observado ritmos circadianos en la ejecución ante muy diversas tareas. Por ejemplo en el tiempo de reacción serial de elección, en tareas de vigilancia (D’Reaux, Neumann \& Rhymer, 2000; Kraemer et al., 2000), en pruebas de cancelación, en la cantidad de dígitos que se pueden repetir, en operaciones aritméticas (Shapiro, Blake, Fossey \& Adams, 1990), en tareas de memoria (Folkard \& Monk, 1980; Vallar \& Baddeley, 1984), en tareas de búsqueda visual, razonamiento lógico y matemático 
(Natale, Alzan \& Cicogna, 2003), en la percepción del tiempo (Kuriyama et al., 2003), en la estimación de la hora del día (Campbell, Murphy \& Boothroyd, 2001), en una tarea que simula la conducción de un automóvil (Lenne, Triggs \& Redman, 1997), en una tarea psicomotora (tiempo de reacción ante un estímulo visual) (Blatter et al., 2006), en la velocidad manual, la búsqueda serial, el razonamiento verbal, una tarea de memoria de trabajo (Monk, Buysse, Reynolds III \& Kupfer, 1998), en una tarea de razonamiento lógico (Monk \& Carrier, 1997), en los componentes de la atención (Valdez et al., 2005), en los componentes de la memoria de trabajo (Ramírez et al., 2006), en tareas manuales, razonamiento verbal, tareas de búsqueda serial (Monk \& Carrier, 1998), una tarea de símbolos y dígitos y en una tarea de evocación (Wright, Hull \& Czeisler, 2002). En la mayor parte de los estudios mencionados se ha encontrado una correlación entre el ritmo circadiano en la temperatura corporal y las variaciones en la ejecución, los niveles de ejecución bajan cuando la temperatura disminuye (Schmidt, Collette, Cajochen \& Peigneux, 2007).

Es posible que las oscilaciones fisiológicas modulen un proceso cognoscitivo básico, el cual podría afectar la ejecución de todas las tareas mencionadas. Un proceso cognoscitivo crucial para el desempeño de una gran cantidad de tareas es la atención sostenida, la cual consiste en la capacidad para responder de forma eficiente al ambiente por periodos de minutos a horas. Se han usado dos tipos de tareas para medir la atención sostenida: una tarea de vigilancia y una tarea de ejecución continua. En una tarea de vigilancia se presentan estímulos de forma infrecuente, por ejemplo en cada 5 o más segundos (Dinges \& Powell, 1985); mientras que en una tarea de ejecución continua los estímulos se presentan cada segundo (Riccio, Reynolds, Lowe \& Moore, 2002). Muchos trabajos han encontrado ritmos circadianos en la atención sostenida por medio de tareas de vigilancia, pero muy pocos trabajos han usado una tarea de ejecución continua (Schmidt, Collette, Cajochen \& Peigneux, 2007). El uso de estimulación frecuente en una tarea de ejecución continua mejora la ejecución, probablemente debido a que induce un estado de alerta (Davies \& Parasuraman, 1982; Warm, 1984). En el presente trabajo se pretende analizar el efecto de la frecuencia de los estímulos sobre los ritmos circadianos en la eficiencia para responder ante una tarea de ejecución continua. En la noche disminuye la ejecución, por lo que un mayor alertamiento, inducido por una mayor frecuencia en los estímulos, podría hacer que la ejecución se mantuviese a niveles altos durante la noche. Es necesario analizar si el efecto de la frecuencia de los estímulos ocurre en la ejecución total ante la tarea, así como en la eficiencia para responder a cada estímulo. No existen trabajos publicados sobre este posible efecto de la frecuencia de estímulos en una tarea de ejecución continua. Para poder observar los ritmos circadianos en la ejecución sin la interferencia de los sincronizadores, en el presente trabajo se usó un "protocolo de rutina constante". 


\section{MÉTODO}

\section{Participantes}

Se registraron 3 mujeres voluntarias, de 17, 18 y 19 años de edad, estudiantes universitarias, que asistían a clases de lunes a viernes con un horario de 07:00-13:30 h, sin actividades programadas durante las tardes y los fines de semana, sin trastornos graves de salud o del sueño, que no se encontraban tomando medicamentos que afectan el sistema nervioso central. Cada participante firmó una carta de consentimiento voluntario, la cual fue firmada también por los padres de la participante que era menor de edad. El proyecto de investigación fue aprobado por un comité académico de la universidad y se llevó a cabo de acuerdo con los principios de la declaración de Helsinki para la investigación con humanos.

\section{Instrumentos}

Se usó una cámara de aislamiento térmico, acústico y de la iluminación solar. En esta cámara se mantuvo la temperatura ambiental en $24 \pm 1^{\circ} \mathrm{C}$. La iluminación se mantuvo por debajo de 300 lux, medida al nivel de los ojos de las participantes. Para la presentación de los estímulos y el registro de la respuesta de las participantes, se usó una computadora, los estímulos aparecieron en un monitor de 14" con una resolución de 800 X600 pixeles. Se utilizó también un termómetro rectal marca Steriprobe 491B conectado a un Mini-Logger 2000 (Philips Respironics), aparato que registró y almacenó la temperatura rectal cada minuto, la información almacenada se transmitió posteriormente a la computadora. Se usaron 4 cuestionarios: de datos generales (edad, salud en general, medicamentos que ingiere); de trastornos del sueño (Téllez, 1998); un diario del dormir (Valdez, Ramírez \& Téllez, 1998) y una versión en español de la escala de madrugadores-trasnochadores (Valdez, Ramírez \& Téllez, 1998).

Para medir la atención sostenida se usó una prueba de ejecución continua (Riccio, Reynolds, Lowe \& Moore, 2002; Valdez et al., 2005) que consistió en lo siguiente: se presentaban por medio de la computadora números de un dígito, las participantes tenían que presionar la tecla "1" ante cualquier número (excepto 9), presionar la tecla "2" ante el 9 y presionar la tecla "3" solamente cuando aparecía un 4 después del 9 . Esta prueba constó de 27 bloques de 20 estímulos cada uno: cada condición tenía la siguiente frecuencia: "número diferente de 9" se presentaba 14 veces (70\%), "número 9" se presentaba 4 veces $(20 \%)$ y "número 4 después de 9 " se presentaba 2 veces $(10 \%)$. La duración de cada estímulo fue de 100 milisegundos (ms), el intervalo entre estímulos variaba alrededor de $1200 \mathrm{~ms}(1000,1100,1200,1300$ y 1400 $\mathrm{ms}$ ). Los estímulos dentro de cada bloque se presentaron de forma aleatoria, nunca se presentaron dos dígitos iguales consecutivos. Los estímulos fueron 
números con un tipo de letra Arial, tamaño 60 , que se presentaron en un monitor de computadora de 14" colocado a $60 \mathrm{~cm}$ de distancia enfrente de la participante. La prueba incluyó un total de 540 estímulos, la duración total fue de 11 minutos y 42 segundos.

\section{Procedimiento}

Inicialmente cada participante firmó la carta de consentimiento de participación voluntaria. Se aplicaron los cuestionarios de datos generales y de trastornos del sueño. Solamente se admitieron en el estudio las personas que no tenían un problema de salud grave, que no estuvieran tomando medicamentos que tuviesen efectos sobre el sistema nervioso y que no tuvieran dificultades en el dormir. Las participantes que se admitieron contestaron la escala de madrugadores-trasnochadores; llevaron a cabo un registro diario de las características del ciclo de vigilia-sueño, durante un período de 11 días seguidos que inició en jueves; además se registró su consumo de alimentos durante 2 días, con lo que se calculó la ingesta de calorías diarias. El promedio de ingesta calórica diaria fue de 3,130; 2,650 y 1,920 kilocalorías (Kcal), en cada una de las participantes.

Cada participante recibió instrucciones y entrenamiento en la prueba de ejecución continua un día antes de acudir a la sesión de registro. Las instrucciones incluyeron abstenerse de consumir alimentos en forma excesiva o con muchas calorías, hacer cualquier otra forma de restricción en la dieta, consumir alcohol, café o cualquier tipo de droga durante 24 horas antes de la sesión de registro. Todas las participantes afirmaron que cumplieron con esta solicitud.

Las participantes acudieron al laboratorio a las $12: 00 \mathrm{~h}$ para el registro en el protocolo de rutina constante; sin embargo, las participantes 1 y 2 se empezaron a registrar a las $14: 00 \mathrm{~h}$ y la participante 3 se empezó a registrar a las 12:00 h. El tiempo total de registro para las participantes fue de al menos $25 \mathrm{~h}$. La primera hora de registro se consideró como práctica, por lo que no se incluyó en el análisis de datos.

Durante la sesión de registro no se permitió el consumo de café, tabaco, ni drogas. Se registró de forma continua su temperatura rectal, así como las respuestas ante una tarea de ejecución continua. Cada registro duraba aproximadamente 20 minutos, seguido por un período de descanso. Como parte del "protocolo de rutina constante", se llevó a cabo un control de la postura corporal, manteniendo a las participantes acostadas en un sillón con una inclinación de $45^{\circ}$ sobre el eje horizontal, con la cabeza elevada con respecto a los pies, excepto por breves períodos cuando tenían que ir al baño. Se llevó a cabo un control de la ingesta calórica, para lo cual cada hora (durante los periodos de descanso) las participantes ingerían una porción de alimento equivalente a la fracción correspondiente a dividir el total de calorías 
de ingesta diaria entre 24 horas. Las porciones por hora de cada participante fueron: 130, 110 y $80 \mathrm{Kcal}$. Con el propósito de evitar posibles variaciones en el comportamiento vinculadas con el ciclo menstrual, ninguna participante se registró 5 días antes a 5 días después de la menstruación. Las participantes se registraron en la siguiente fase de su ciclo menstrual: la participante 1 se registró el día 8 de un ciclo regular de 30 días, posiblemente en la fase folicular; la participante 2 se registró el día 20 de un ciclo regular de 28 a 30 días, posiblemente en la fase luteínica; la participante 3 se registró el día 48 de un ciclo irregular de 30 a 60 días, posiblemente en la fase luteínica.

\section{Análisis de datos}

Para el análisis de los datos de temperatura rectal se usó la técnica cosinor, que permite determinar si los datos de esta variable se ajustan a un ciclo de $24 \mathrm{~h}$ (Minors \& Waterhouse, 1988). Durante una sesión de $25 \mathrm{~h}$ de registro la persona se mantiene activa y despierta, por lo que ocurre fatiga y privación del dormir. Estas condiciones producen una reducción en el rendimiento, la cual se manifiesta como una regresión lineal negativa en la ejecución. Para separar las variaciones circadianas de los posibles efectos de la fatiga y la privación del dormir, se utilizó la técnica de eliminación de la tendencia lineal, la cual consiste en restar la regresión lineal a los datos de cada variable por cada hora de registro, este análisis se llevó a cabo con los datos de cada participante. Para el análisis de las relaciones entre las respuestas a las teclas se calcularon los coeficientes de correlación producto-momento de Pearson. Se usó también la técnica de correlación cruzada, la cual permite identificar la relación de fase de una variable de prueba con respecto a otra que se toma como criterio (Diggle, 1990). Esta técnica consiste en obtener los coeficientes de correlación que resultan de cambiar la alineación de los datos de la variable de prueba con respecto a la variable criterio. La correlación alcanza un valor más alto cuando ambas variables se encuentran en fase. Se obtuvieron los valores de correlación cruzada para cada una de las variables de ejecución (variables de prueba) con respecto a la temperatura rectal (variable criterio).

\section{RESULTADOS}

De acuerdo con la autoevaluación de la fase circadiana, las participantes tuvieron una calificación de 41, 49 y 53 puntos, por lo que se pueden clasificar como cronotipo intermedio (sin tendencia madrugadora o trasnochadora). Se observaron diferencias en el nivel absoluto de la temperatura rectal de las participantes, los valores promedio (mesor) de temperatura en cada una de las participantes fueron: $36.64,37.29$ y 36.70 grados Celsius, lo cual puede 
estar vinculado a las variaciones en la temperatura corporal que se observan durante el ciclo menstrual en las mujeres. A pesar de estas diferencias en el nivel absoluto de temperatura, se observaron oscilaciones circadianas en la temperatura corporal, los valores más altos ocurrieron en la tarde (alrededor de las 18:00 - 20:00 h) y los valores más bajos en la madrugada (alrededor de las 02:00 - 04:00 h) (Figuras 1-3). En el análisis cosinor, se observó un ajuste significativo de los datos del registro de la temperatura corporal de cada una de las participantes, a una curva sinusoidal con un período de $24 \mathrm{~h}$. Los valores de ajuste a un periodo de $24 \mathrm{~h}$, fueron de $83.25,75.83,90.46 \%$ en cada una de las participantes. De acuerdo con el ajuste cosinor, la acrofase (momento en que ocurre el valor más alto) ocurrió a las 15:27, 14:36 y 15:34 (horas:minutos) de cada participante. Se observaron diferencias significativas en el ciclo de vigilia-sueño entre semana comparado con el fin de semana. Entre semana las participantes se acostaban a las $24: 41,23: 57$ y $23: 19$ horas del día y se levantaban a las 06:16, 05:59 y 04:48 horas del día, por lo que dormían 5:45, 6:03 y 5:27 horas. En cambio, el fin de semana se acostaban a las $25: 45,24: 56$ y 23:51 horas del día y se levantaban a las 10:04, 08:39 y 08:53 horas del día, por lo que dormían 8:19, 8:04 y 9:01 horas. En resumen, el fin de semana las participantes se acostaron y se levantaron más tarde, además durmieron más tiempo. No se observaron relaciones entre la fase de la temperatura, el ciclo de vigilia-sueño y el cronotipo medido a partir de la escala de madrugadores-trasnochadores.

En la prueba de ejecución continua, la eficiencia para responder (porcentaje de respuestas correctas) mostró un deterioro en la ejecución durante la sesión de registro. La baja en la ejecución se puede estimar por medio de la regresión lineal, cuyos valores para cada participante fueron de: $-0.67,-0.65$ y -0.74. Sin embargo, al eliminar la tendencia lineal (restando la regresión lineal) se observaron variaciones durante las 24 horas del día. La eficiencia para responder aumentó durante el día hasta alcanzar valores máximos (acrofases) alrededor de las 22:00-24:00 h, mientras que disminuyó en la noche hasta alcanzar valores mínimos (batifases) en la madrugada (alrededor de las 04:00-07:00 h). La disminución en la eficiencia y el aumento en el tiempo de reacción ocurrieron cerca del momento en que bajó la temperatura rectal. Se observaron variaciones circadianas en todas las tareas, tanto en las respuestas a la tecla "1" (frecuencia de los estímulos: $70 \%$ ), como a la tecla "2" (frecuencia de los estímulos: $20 \%$ ) y "3" (frecuencia de los estímulos: $10 \%$ ). La ejecución ante cada una de las teclas siguió un patrón similar a la eficiencia general, el porcentaje de respuestas correctas aumentó durante el día, mientras que disminuyó en la madrugada (Figuras 1-3). Se analizaron los errores y las omisiones, sin embargo no se presentan esos datos ya que siguen un patrón recíproco con respecto al porcentaje de respuestas correctas, esto es, los errores y omisiones aumentaban cuando disminuía la 
eficiencia. Se observaron correlaciones muy altas en la ejecución de cada participante a las distintas teclas. Todas las correlaciones fueron superiores a $0.89(p<0.01)$.

Con el análisis de correlación cruzada se observó un retraso de fase del porcentaje de respuestas correctas (totales y por cada una de las teclas) con respecto a la temperatura rectal, en todas las participantes. Para el total de respuestas correctas el retraso fue de $5(r=0.61), 2(r=0.70)$ y $3(r=0.90)$ horas en cada participante. Para las respuestas a la tecla 1 el retraso fue de $5(r=0.61), 2(r=0.70)$ y $2(r=0.90)$ horas para cada participante. Para las respuestas a la tecla 2 el retraso fue de $5(r=0.63), 2(r=0.70)$ y $3(r=0.88)$ horas para cada participante. Para las respuestas a la tecla 3 el retraso fue de 5 (0.62), $1(r=0.65)$ y $3(r=0.84)$ horas para cada participante. El tiempo de reacción no mostró una fase consistente con respecto a la temperatura rectal, en la participante 1 el tiempo de reacción estaba en fase con la temperatura rectal $(r=-0.79)$, en la participante 2 se observó un adelanto de fase de una hora $(r=-0.81)$ y en la participante 3 se observó un retraso de fase de 4 horas $(r=-0.77)$. Todos los coeficientes de correlación mencionados son estadísticamente significativos $(p<0.05)$. Los resultados de la técnica de correlación cruzada toman en cuenta todos los valores de las curvas de temperatura y de ejecución, por lo que pueden no coincidir exactamente con la diferencia de fase que se observa visualmente en las gráficas (Figuras 1-3). 


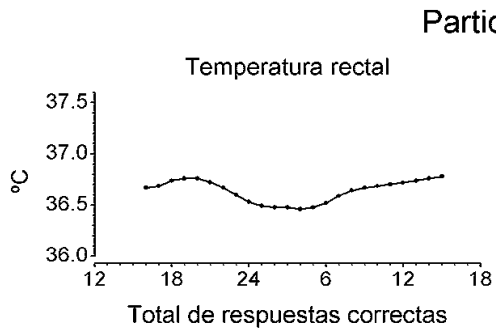

Participante 1
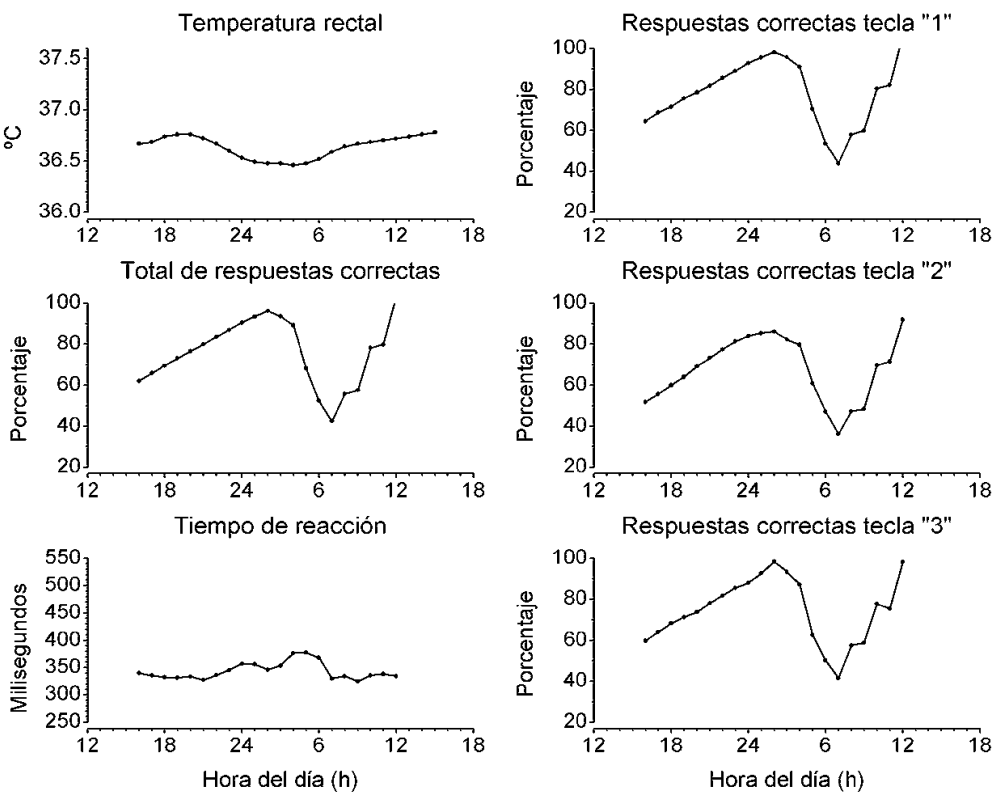

Figura 1. Resultados de la participante 1. Temperatura rectal y eficiencia para responder en la prueba de ejecución continua. Las gráficas que se encuentran a la izquierda representan la temperatura rectal, la eficiencia general (total de respuestas correcta) y el tiempo de reacción. Las gráficas que se encuentran a la derecha representan la eficiencia para responder ante cada una de las teclas, las frecuencias de estímulo para cada tecla fueron de 70 , 20 y $10 \%$, respectivamente. 

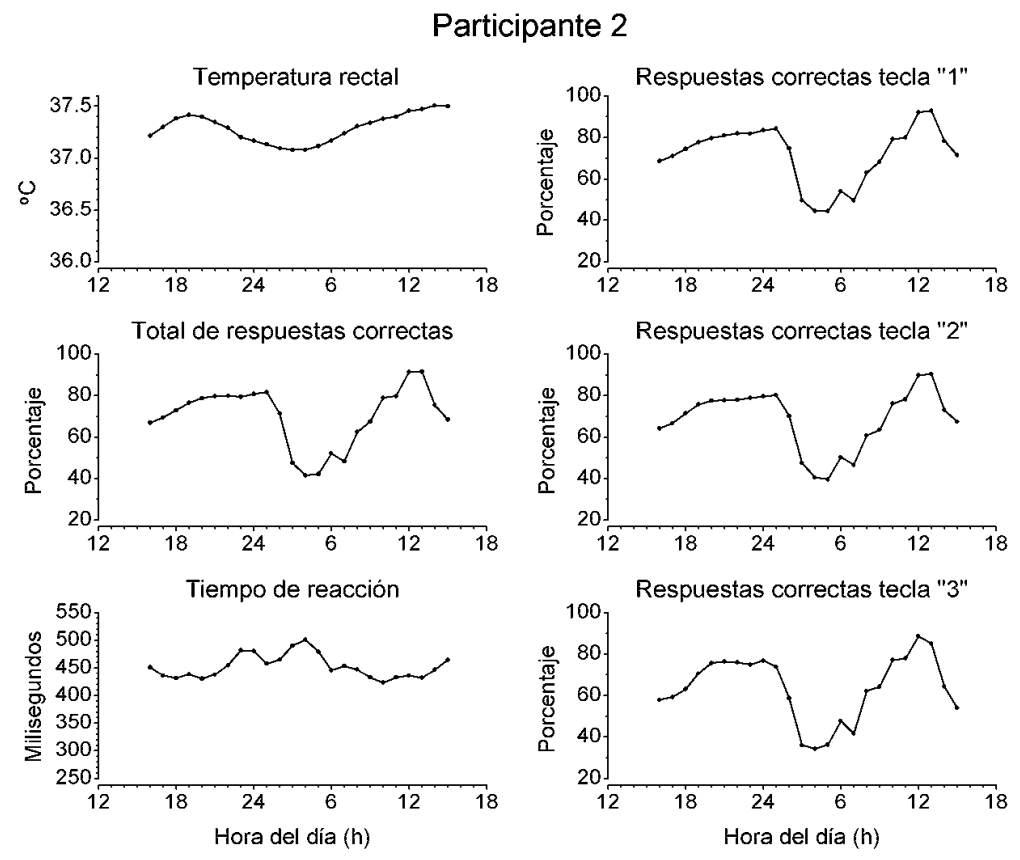

Figura 2. Resultados de la participante 2. Temperatura rectal y eficiencia para responder en la prueba de ejecución continua. Las gráficas que se encuentran a la izquierda representan la temperatura rectal, la eficiencia general (total de respuestas correcta) y el tiempo de reacción. Las gráficas que se encuentran a la derecha representan la eficiencia para responder ante cada una de las teclas, las frecuencias de estímulo para cada tecla fueron de 70 , 20 y $10 \%$, respectivamente. 


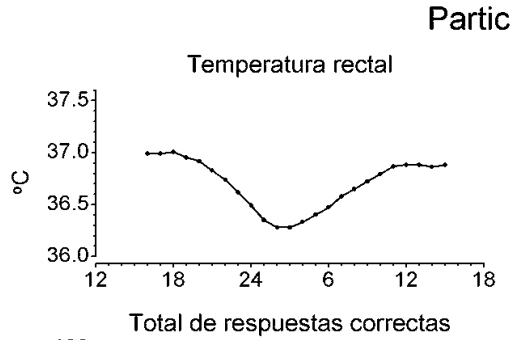

Participante 3
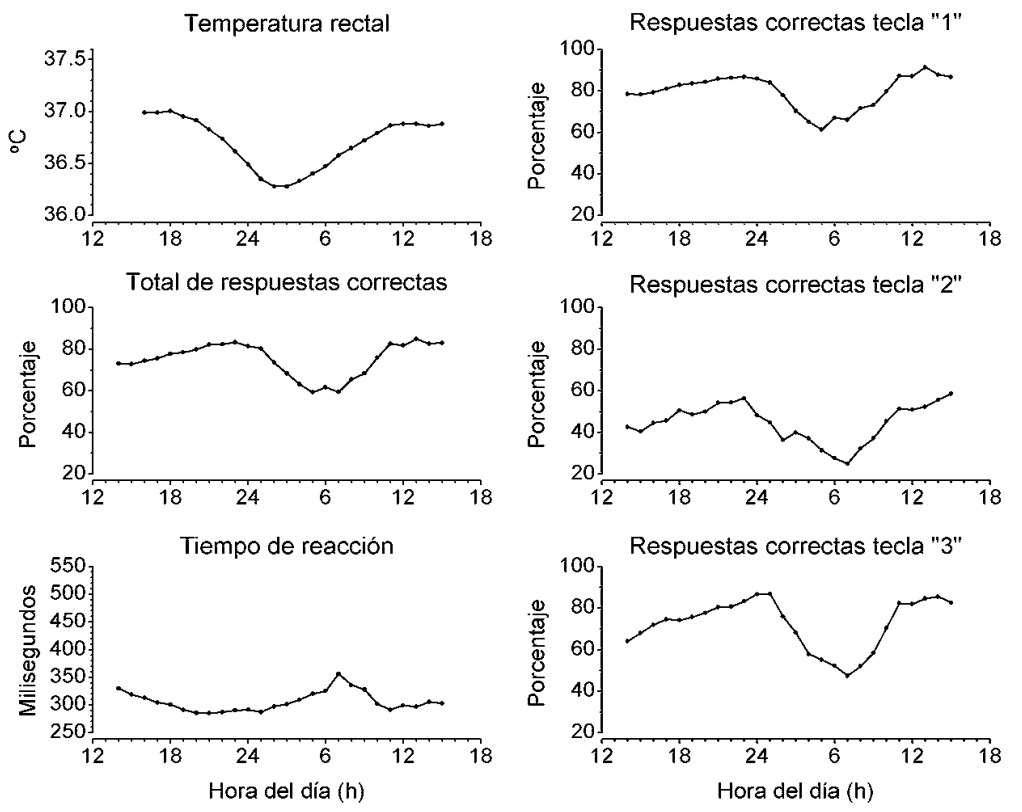

Figura 3. Resultados de la participante 3. Temperatura rectal y eficiencia para responder en la prueba de ejecución continua. Las gráficas que se encuentran a la izquierda representan la temperatura rectal, la eficiencia general (total de respuestas correcta) y el tiempo de reacción. Las gráficas que se encuentran a la derecha representan la eficiencia para responder ante cada una de las teclas, las frecuencias de estímulo para cada tecla fueron de 70 , 20 y $10 \%$, respectivamente. 


\section{DISCUSIÓN}

En el presente trabajo se observaron ritmos circadianos en la temperatura corporal y en la eficiencia para responder en una prueba de ejecución continua, la cual mide la atención sostenida. Además, se observó que en el momento en que empezó a bajar la temperatura corporal disminuyó la eficiencia para responder a cada una de las teclas y aumentó el tiempo de reacción. Estos resultados pueden interpretarse como apoyo a la teoría de Kleitman, de acuerdo con la cual los cambios en el metabolismo corporal producen variaciones en la ejecución (Dement \& Kleitman, 1957). Sin embargo, los datos de la relación de fase entre la temperatura y la ejecución no concuerdan de forma exacta con la teoría de Kleitman. Aunque la temperatura corporal y la ejecución tienden a concordar en el tiempo, los cambios en la ejecución ocurren con un retraso de fase de 2 a 5 horas con respecto a la temperatura corporal. Se pueden plantear tres posibles explicaciones de este retraso de fase en la ejecución: primera, los cambios en la temperatura inducen efectos directos pero lentos en la ejecución; segunda, la temperatura corporal modula la ejecución a través de otra variable que no se registró en este estudio; tercera, existen ritmos circadianos independientes en la temperatura corporal y en la ejecución, por lo que cada ritmo tiene su propia fase. Se requiere diseñar otro tipo de estudios para analizar cuales factores intervienen en el retraso de fase de la ejecución con respecto a la temperatura corporal.

Las variaciones circadianas en la eficiencia ante una tarea de ejecución continua concuerdan con los resultados que se obtienen al usar tareas de vigilancia. Esto implica que los ritmos circadianos en la atención sostenida prevalecen independientemente de la frecuencia de los estímulos que tenga la tarea. Acorde con esta conclusión, se observaron ritmos circadianos no solamente en la eficiencia general, sino en la eficiencia para responder a cada uno de los estímulos de la tarea, a pesar de que cada uno de ellos se presentó con diferente frecuencia.

Los resultados de este estudio demuestran que el comportamiento humano presenta variaciones circadianas, esto es, la ejecución es diferente a distintas horas del día, tanto en la eficiencia general como en la eficiencia para responder ante estímulos con diferente frecuencia. En consecuencia, es necesario tomar en cuenta los ritmos circadianos en el análisis de la conducta, tanto de los animales como del ser humano.

Las variaciones circadianas que se observaron en este trabajo tienen repercusiones sobre muchas áreas aplicadas. La prueba de ejecución continua que se usó en el presente trabajo es similar a las versiones que se usan en el campo profesional que se conoce como neuropsicología clínica (Lezak, Howieson \& Loring, 2004). Este tipo de pruebas se usan para identificar una reducción en la capacidad para responder en pacientes con daño o trastor- 
nos cerebrales. Generalmente no se toma en cuenta la hora del día en que se lleva a cabo la evaluación del paciente. Los resultados de este estudio demuestran que la ejecución en esta prueba es diferente durante el transcurso del día, por lo que es necesario tomar en cuenta la hora en que se lleva a cabo la evaluación para determinar si existe una deficiencia en la capacidad de respuesta de los pacientes con respecto a los valores que se producen en diferentes momentos del día. En resumen, es importante tomar en cuenta que existen ritmos circadianos en la fisiología y el comportamiento de los animales y el ser humano, tanto en la investigación del comportamiento, como en el análisis de los trastornos en el comportamiento.

Uno de los campos donde este hallazgo tiene también implicaciones es en el trabajo, tanto en el trabajo nocturno, como en los turnos rotatorio y diurno (Tucker, Folkard \& Macdonald, 2003). Cuando una persona tiene que trabajar una jornada prolongada, lo más probable es que su ejecución se deteriore progresivamente (Valdez, 1988). Esta reducción es mayor durante la madrugada y primeras horas de la mañana (Valdez, Ramírez, García \& Talamantes, 2008). Podemos poner como ejemplo a un conductor, ya sea de un avión, ferrocarril, un automóvil, un autobús de pasajeros o un vehículo de transporte. Entre más tiempo permanezca trabajando de forma continua, peor será su ejecución, pero en la madrugada y en las primeras horas de la mañana, cuando le disminuye la temperatura corporal, se reducirá su rendimiento (Valdez, Reilly \& Waterhouse, 2008). Podemos prever que en estas condiciones la persona seguirá conduciendo, pero existe una mayor probabilidad de que suceda un accidente si ocurre cualquier evento inesperado y la persona tiene que dar respuestas específicas.

\section{REFERENCIAS}

Åkerstedt, T., Knutsson, A., Westerholm, P., Theorell, T., Alfredsson, L. \& Kecklund, G. (2004). Mental fatigue, work and sleep. Journal of Psychosomatic Research, $57,427-433$

Baldwin, B. A. \& Parrott, R. F. (1979). Studies on intracranial electrical self-stimulation in pigs in relation to ingestive and exploratory behaviour. Physiology \& Behavior, 22(4), 723-730.

Blatter, K., Graw, P., Munch, M., Knoblauch, V., Wirz-Justice, A. \& Cajochen, C. (2006). Gender and age differences in psychomotor vigilance performance under differential sleep pressure conditions. Behavioural Brain Research, 168(2), 312-317.

Borbély, A. A. (1982). A two process model of sleep regulation. Human Neurobiology, 1(3), 195-204.

Campbell, S. S., Murphy, P. J. \& Boothroyd, C. E. (2001). Long-term time estimation is influenced by circadian phase. Physiology \& Behavior, 72(4), 589-593. 
Carrier, J. \& Monk, T. H. (2000). Circadian rhythms of performance: new trends. Chronobiology International, 17(6), 719-732.

Colquhoun, W. P. (1971). Circadian variations in mental efficiency. En W. P. Colquhoun (Ed.), Biological Rhythms and Human Performance (pp. 39-107). London: Academic Press.

Davies, D. R. y Parasuraman, R. (1982). The Psychology of Vigilance. London: Academic Press.

D'Reaux, R. A., Neumann, C. S. \& Rhymer, K. N. (2000). Time of day of testing and neuropsychological performance of schizophrenic patients and healthy controls. Schizophrenia Research, 45, 157-167.

Dement, W. \& Kleitman, N. (1957). Cyclic variations in EEG during sleep and their relation to eye movements, body motility, and dreaming. Electroencephalography and Clinical Neurophysiology, 9(4), 673-690.

Diggle, P. (1990). Time series: a bio-statistical introduction. New York: Oxford University Press.

Dinges, D. F. \& Powell, J. W. (1985). Microcomputer analyses of performance on a portable, simple visual RT task during sustained operations. Behavior Research Methods, Instruments, \& Computers, 17, 652-655.

Folkard, S. \& Monk, T. H. (1980). Circadian rhythms in human memory. British Journal of Psychology, 71, 295-307.

Hanneman, S. K. (2001). Measuring circadian temperature rhythm. Biological Research for Nursing, 2(4), 236-248.

Kleitman, N. (1963). Sleep and wakefulness. Chicago: University of Chicago Press.

Koukkari, W. L. y Sothern, R. B. (2006). Introducing biological rhythms : a primer on the temporal organization of life, with implications for health, society, reproduction and the natural environment. New York: Springer.

Kraemer, S., Danker-Hopfe, H., Dorn, H., Schmidt, A., Ehlert, I. \& Herrmann, W. M. (2000). Time-of-day variations of indicators of attention: performance, physiologic parameters, and self-assessment of sleepiness. Biological Psychiatry, 48(11), 1069-1080.

Kuriyama, K., Uchiyama, M., Suzuky, H., Tagaya, H., Ozaki, A., Aritake, S., et al. (2003). Circadian fluctuation of time perception in healthy human subjects. Neuroscience Research, 46, 23-31.

Lavie, P. (1980). The search for cycles in mental performance from Lombarb to Kleitman. Chronobiologia, 7(2), 247-256.

Lenne, M. G., Triggs, T. J. \& Redman, J. R. (1997). Time of day variations in driving performance. Accident, Analysis and Prevention, 29(4), 431-437.

Lezak, M. D., Howieson, D. B. y Loring, D. W. (2004). Neuropsychological assessment (4th ed.). New York: Oxford University Press.

Luce, G. G. (1971). Biological rhythms in human and animal physiology. New York: Dover Publications.

Minors, D. S. \& Waterhouse, J. M. (1988). Mathematical and statistical analysis of circadian rhythms. Psychoneuroendocrinology, 13(6), 443-444.

Monk, T. H., Buysse, D. J., Reynolds III, C. F. \& Kupfer, D. J. (1998). Endogenous circadian performance rhythms-relationship to temperature, cortisol, melatonin, 
mood and alertness. En Y. Touitou (Ed.), Biological Clocks, Mechanisms and Applications (pp. 557-562). New York: Elsevier Science BV.

Monk, T. H. \& Carrier, J. (1997). Speed of mental processing in the middle of the night. Sleep, 20(6), 399-401.

Monk, T. H. \& Carrier, J. (1998). A parallelism between human body temperature and performance independent of the endogenous circadian pacemaker. Journal of Biological Rhythms, 13(2), 113-122.

Moore-Ede, M. C., Sulzman, F. M. \& Fuller, C. A. (1982). The clocks that time us. Cambridge: Harvard University Press.

Natale, V., Alzan, A. \& Cicogna, P. C. (2003). Cognitive efficiency and circadian typologies: a diurnal study. Personality and Individual Differences, 35(5), 1089-1105.

Palmer, J. D. (1976). An introduction to biological rhythms. New York: Academic Press.

Ralph, M. R., Ko, C. H., Antoniadis, E. A., Seco, P., Irani, F., Presta, C., et al. (2002). The significance of circadian phase for performance on a reward-based learning task in hamsters. Behavioural Brain Research, 136(1), 179-184.

Ramírez, C., Talamantes, J., García, A., Morales, M., Valdez, P. \& Menna-Barreto, L. (2006). Circadian rhythms in phonological and visuospatial storage components of working memory. Biological Rhythm Research, 37(5), 433-441.

Riccio, C. A., Reynolds, C. R., Lowe, P. \& Moore, J. J. (2002). The continuous performance test: a window on the neural substrates for attention? Archives of Clinical Neuropsychology, 17(3), 235-272.

Schmidt, C., Collette, F., Cajochen, C. \& Peigneux, P. (2007). A time to think: Circadian rhythms in human cognition. Cognitive Neuropsychology, 24(7), 755-789.

Shapiro, C. M., Blake, F., Fossey, E. \& Adams, B. (1990). Daylight saving time in psychiatric illness. Journal of Affective Disorders, 19(3), 177-181.

Téllez, A. (1998). Trastornos del sueño: diagnóstico y tratamiento. México: Trillas.

Touitou, Y. (1998). Biological clocks. Mechanisms and applications. Amsterdam: Elsevier.

Tucker, P., Folkard, S. \& Macdonald, I. (2003). Rest breaks and accident risk. Lancet, 361(9358), 680.

Valdez, P. (1988). Ritmos circadianos y conducta. En E. C. Valcárcel (Ed.), La Neuropsicología, una nueva rama en el conocimiento psicológico (pp. 167-206). La Habana: ENPES.

Valdez, P., Pérez, J. C., Galarza, J. \& Ramírez, A. (1986). Ritmos circadianos y ultradianos en la ingestión de agua en ratas. Revista Mexicana de Análisis de la Conducta, 12(2), 137-145.

Valdez, P., Ramírez, C., García, A., \& Talamantes, J. (2008). Los cambios de la atención a lo largo del día. Ciencia, 59 (1), 14-23.

Valdez, P., Ramírez, C., García, A., Talamantes, J., Armijo, P. \& Borrani, J. (2005). Circadian rhythms in components of attention. Biological Rhythm Research, 36(1/2), 57-65.

Valdez, P., Ramírez, C. \& Téllez, A. (1998). Alteraciones del ciclo dormir-vigilia. En A. Téllez (Ed.), Trastornos del sueño: diagnóstico y tratamiento (pp. 193-230). México: Trillas. 
Valdez, P., Reilly, T. \& Waterhouse, J. (2008). Rhythms of mental performance. Mind, Brain and Education, 2(1), 7-16.

Vallar, G. \& Baddeley, A. (1984). Fractionation of working memory: neuropsychological evidence for a phonological short-term store. Journal of Verbal Learning and Verbal Behavior, 23, 151-161.

Warm, J. S. (1984). Sustained attention in human performance. New York: John Wiley \& Sons.

Wright, K. P., Jr., Hull, J. T. \& Czeisler, C. A. (2002). Relationship between alertness, performance, and body temperature in humans. American Journal of Physiology. Regulatory, Integrative and Comparative Physiology, 283(6), 1370-1377. 